\title{
AN ATTEMPT TO INDUCE TOLERANCE WITH INFUSION OF DONOR BONE MARROW IN ORGAN ALLOGRAFT RECIPIENTS*
}

\author{
Abdul S. Rao, ${ }^{1}$ D. Phil, ${ }^{2,3}$ Paulo Fontes, ${ }^{2}$ Anand Iyengar, ${ }^{2}$ \\ Adelouahab Aitouche, ${ }^{2}$ Ron Shapiro, ${ }^{2}$ Adriana Zeevi, ${ }^{3}$ Forrest Dodson, ${ }^{2}$ \\ Robert Corry, ${ }^{2}$ Cristiana Rastellini, ${ }^{2}$ John J. Fung, ${ }^{2}$ and Thomas E. Starzl ${ }^{2}$ \\ 'Thomas E. Starzl Transplantation Institute \\ ${ }^{2}$ Department of Surgery and \\ ${ }^{3}$ Department of Pathology \\ University of Pittsburgh Medical Center \\ Pittsburgh, Pennsylvania
}

\section{THE ROLE OF PASSENGER LEUKOCYTES IN ALLOGRAFT REJECTION}

Early observations by Snell and Steinmuller provided unequivocal evidence that nonparenchymal cells, resident within the organ play an important role in allograft rejection ${ }^{1-3}$.

However, despite these findings and the subsequent employment of the term "passenger leukocytes" to illustrate their migratory capacity ${ }^{4}$, identity of the donor cells involved in allograft rejection (at that time) was nevertheless enigmatic. These observations nonetheless had two unique corollaries for transplantation (Tx). Firstly, that the severity of rejection might be mitigated by functionally modulating or depleting donor leukocytes prior to organ $\mathrm{tx}$, and secondly, that the majority of the transplanted tissue is non-immunogenic and may therefore play an infinitesimal role in graft rejection. The realization of the importance of these findings prompted the initiation of numerous studies intended to modulate the immune responses by manipulating resident non-parenchymal cells prior to transplantation ${ }^{5-7}$. In spite of the novelty of these experiments, the success was however, variable.

\subsection{Importance of Dendritic Cells (DC) in Primary Immune Responses}

Whilst the role of passenger leukocytes in initiating allograft rejection was undeniable, the identity of the cell(s) which instigated this immune response was however,

* Aided by Project Grant No. AI 40329 and DK 29961 from the National Institute of Health, Bethesda, Maryland. 
equivocal. Given their unique capacity to present antigens to naive $T$ cells, it was postulated that perhaps cells of dendritic leukocyte lineage may play a vital role in allosensitization $^{8}$. Supporting evidence for the latter argument came from the initial studies by Lechler and Batchelor, who demonstrated that allogeneic kidneys were indefinitely accepted in rodents if they were initially "parked" in an immunosuppressed recipient prior to retransplantation into a secondary naive syngeneic animal ${ }^{9}$. More importantly, the allografts were acutely rejected if DC obtained from donor-strain animals were infused into the secondary recipients indicating that the latter manipulation perhaps, led to the restoration of immunogenicity of the leukocyte-depleted allograft resulting in its prompt rejection. This and numerous subsequent studies ${ }^{10-12}$ have unambiguously established the identity of donor DC as the critical passenger leukocytes primarily responsible for allograft rejection, supporting the concept that their elimination prior to $\mathrm{Tx}$ may possibly culminate in prolonged graft survival.

\subsection{DC in Transplantation Tolerance}

A diametrical yet entirely compatible role for DC has been proposed by recent studies in rodents ${ }^{13,14}$ and sub-human primates ${ }^{15}$. Our laboratory has shown previously that livers transplanted orthotopically across most mouse strain combinations are spontaneously accepted $^{16}$. Interestingly, DC isolated from murine livers exhibit phenotype and function distinctive of that of immature cells, suggesting perhaps that upon migration from the graft into the host, these cells may provide "deviant" signals resulting in induction of donor-specific hyporeactivity rather than allosensitization ${ }^{13}$. It is interesting to note that organs other than livers which are promptly rejected following $T x$ also contain, albeit few, resident immature $\mathrm{DC}^{17}$. It is therefore entirely conceivable that the inherent tolerogenicity uniquely ascribed to murine livers may be contingent on the presence within it an apropos quality and quantity of resident leukocytes bearing an immature phenotype.

\subsection{Chimerism and Its Role in Organ Allograft Acceptance}

The ubiquitous detection of the presence of donor cell chimerism in the tissues of successful long-term human kidney and liver recipients provided irrevocable evidence for the role of microchimerism in Tx tolerance ${ }^{18,19}$. These observations were supported by contemporaneous studies in rodents prompting evolvement of a hypothesis that the establishment of microchimerism by migratory resident leukocytes may play a seminal role in the induction of donor-specific tolerance ${ }^{13,15,20,21}$. Whilst the role of migratory donor cells in the induction of donor-specific hyporeactivity was undeniable, the mechanism responsible for the perpetuation of chimerism and therefore of tolerance, years after organ Tx was nevertheless unclear. However, recent studies in rodents $\mathrm{s}^{22,23}$ and humans ${ }^{24}$, have confirmed the presence of pluripotent stem cells in the interstitium of the grafted organ providing explication for the long-term persistence of donor cell chimerism.

\section{AUGMENTATION OF CHIMERISM IN HUMAN ALLOGRAFT RECIPIENTS}

Recognizing the seminal role played by migratory donor leukocytes in allograft acceptance and the induction of donor-specific tolerance almost four years ago, we embarked on a prospective clinical trial to augment the phenomenon in organ recipients by 
perioperative donor bone marrow (BM) infusion. In addition to verifying the safety of this procedure, three long-term goals of this study were to: (i) prevent delayed graft rejection, (ii) abrogate or mitigate chronic rejection and (iii) to decrease drug-dependence. Since June 1992, 189 recipients of liver $(n=55)$, kidney $(n=36)$, kidney + pancreas $(n=33)$, kidney + islets $(n=7)$, heart $(n=24)$, lungs $(n=18)$, small bowel $(n=13)$ and multi-organ $(n=3)$ have received a single peri-operative infusion of $3-6 \times 10^{8} \mathrm{BM}$ cells $/ \mathrm{kg}$ body weight. Additionally, subsequent to the implementation of a modified protocol in April 1996, ten recipients of liver $(n=5)$, kidney $(n=4)$, kidney + delayed islets $(n=1)$ have received multiple infusions of $1 \times 10^{8}$ cells $/ \mathrm{kg}$ body weight/day for five consecutive days (day $0-4$ ) post-Tx.

Immunosuppression (IS) was with tacrolimus and steroids; CellCept was added to this regimen in 32 study and 15 control patients. BM was isolated from the vertebral bodies (VB) of cadaveric donors by a method described previously ${ }^{25}$ and infused intravenously without cryopreservation. The recipients were not conditioned nor was the BM modified prior to infusion. The unavailability of consent to retrieve VB from the cadaveric donors resulted in accrual of 115 recipients of liver $(n=32)$, kidney $(n=19)$, kidney + pancreas $(n=20)$, kidney + delayed islets $(n=2)$, heart $(n=20)$, lung $(n=10)$, small bowel $(n=7)$ and multiorgan $(n=1)$ who were followed as contemporaneous controls. Episodes of acute rejection were treated with dose adjustments of routine IS, whereas OKT3 was reserved for the treatment of steroid-resistant rejection.

\subsection{In Vitro Monitoring}

Using peripheral blood mononuclear cells (PBMC), the immune status of the recipients was monitored serially by mixed leukocyte reaction (MLR), limiting dilution assay and proliferation against recall antigens (ConA and PHA). Using primers specific for either HLA-allele or the sex-determining region of the Y (SRY) chromosome (in malemfemale recipients), the presence of donor DNA was determined periodically in the study and control patients. Serial quantitative evaluations of donor cell chimerism in a selected cohort of study and control patients were performed using a modified limiting dilution PCR (LDA-PCR) assay. The multilineage character of chimerism was also ascertained by PCR detection of donor DNA in lineage ${ }^{+}$cells sorted from recipients' PBMC. The evidence for the presence of donor DC progenitors and therefore of engraftment was obtained by propagation of recipients' PBMC in rhGM-CSF and rhIL-4-enriched cultures. Subsequent to enrichment for lineage ${ }^{\text {null }} / \mathrm{MHC}$ class II $^{+}$population, the presence of donor DNA within the sorted cells was confirmed by PCR analysis.

\subsection{Clinical Outcome}

The ancillary BM infusion was safe and no complications that could be uniquely attributed to this procedure were witnessed in any of the 199 study patients. All but 18/199 (9\%) of BM-augmented recipients are alive compared to $13 / 115(11 \%)$ of controls, who have died during the course of this follow-up (Table 1). Additionally, grafts in nine study and five control patients have been lost during their successive follow-up (Table 1). It is noteworthy that no deaths or graft losses in the augmented group were related to BM infusion. No evidence of any inimical complication was witnessed in any of the ten patients who have received multiple BM infusion during the course of their follow-up (5-154 days). All of the surviving patients have adequate graft function.

Whilst all patients remain IS-dependent, it is nonetheless interesting to note that steroid-free existence has been achieved in $61 \%$ study and $40 \%$ control patients who are at 
Table 1. BM-Augmented (study) and non-augmented (control) transplant recipients: follow-up and patient graft survival

\begin{tabular}{llccc}
\hline Organs Tx & $n$ & $\begin{array}{c}\text { Follow-up } \\
\text { POD }(\mathrm{X} \pm \mathrm{SD})\end{array}$ & $\begin{array}{c}\text { Patient Survival } \\
(\%)\end{array}$ & $\begin{array}{c}\text { Graft Survival } \\
(\%)\end{array}$ \\
\hline Liver & & & & \\
$\quad$ Study & 60 & $610 \pm 366$ & $53 / 60(88 \%)$ & $51 / 60(85 \%)$ \\
$\quad$ Control & 32 & $798 \pm 354$ & $28 / 32(88 \%)$ & $28 / 32(88 \%)$ \\
Kidney & & & & \\
$\quad$ Study & 81 & $588 \pm 311$ & $80 / 81(99 \%)$ & $75 / 81(93 \%)$ \\
$\quad$ Control & 41 & $617 \pm 311$ & $38 / 41(93 \%)$ & $37 / 41(90 \%)$ \\
Heart & & & & \\
$\quad$ Study & 24 & $481 \pm 330$ & $21 / 24(88 \%)$ & $21 / 24(88 \%)$ \\
Control & 20 & $429 \pm 226$ & $18 / 20(90 \%)$ & $18 / 20(90 \%)$ \\
Lung & & & & \\
Study & 18 & $380 \pm 269$ & $14 / 18(78 \%)$ & $14 / 18(78 \%)$ \\
Control & 10 & $478 \pm 188$ & $8 / 10(80 \%)$ & $8 / 10(80 \%)$ \\
Small Bowel & & & & \\
$\quad$ Study & 13 & $289 \pm 234$ & $10 / 13(77 \%)$ & $9 / 13(69 \%)$ \\
$\quad$ Control & 07 & $302 \pm 219$ & $5 / 7(71 \%)$ & $5 / 7(71 \%)$ \\
Multi-organ & & & & $3 / 3(100 \%)$ \\
$\quad$ Study & 03 & $508 \pm 136$ & $3 / 3(100 \%)$ & $1 / 1(100 \%)$ \\
$\quad$ Control & 01 & 248 & $1 / 1(100 \%)$ & \\
\hline
\end{tabular}

least 12 months post-Tx (figure 1). Equally significant however, is the observations that a statistically higher number of kidney recipients in the study group (71\%) were weaned off steroids as compared to the controls.

2.2.1. Incidence of Acute and Chronic Rejection. The tempo, severity and cumula tive incidence of acute cellular rejection was comparable $(\sim 60 \%)$ in patients in the study and control groups. Graft versus host disease was witnessed in only two (1\%) BM-aug: mented recipients (both of liver) which for its resolution required minor dose adjustments of routine IS. Whilst histopathological changes pathognomonic of chronic rejection have not manifested in any BM-augmented patient, at a comparable duration of follow-up $2 / 7$ (29\%) surviving non-augmented lung recipients have however, exhibited evidence for the development of obliterative bronchiolitis.

\subsubsection{Multilineage Chimerism and Evidence for Infused Donor Cell Engraftment.} The incidence of chimerism was much higher $(94 \%)$ in the BM-augmented patients as compared to the controls (56\%). Additionally, using LDA-PCR, the levels of chimerisr were found to be at least $10-100$ fold higher in a selected cohort of study patients as com: pared to the controls. Furthermore, the presence of donor DNA in sorted lineage ${ }^{+}$cells provided unequivocal evidence of the multilineage nature of donor cell chimerism in BMaugmented patients. It must be emphasized that our ability to identify donor DNA in cul. tured/sorted (lineage ${ }^{+} /$class $\mathrm{II}^{+}$) cells generated from the PBMC of $4 / 5$ evaluated studj patients who were at least one year post-Tx provide sustenance to our claim that infuses marrow may have indeed engrafted.

2.2.3. In Vitro Immune Modulation. A previously established criteria was used to ac cess the extent of donor-specific immune modulation in control and study patients. ${ }^{26} \mathrm{At}$ : two-year follow-up, a higher proportion of BM-augmented liver (50\%) and lung $(60 \%)$ re 


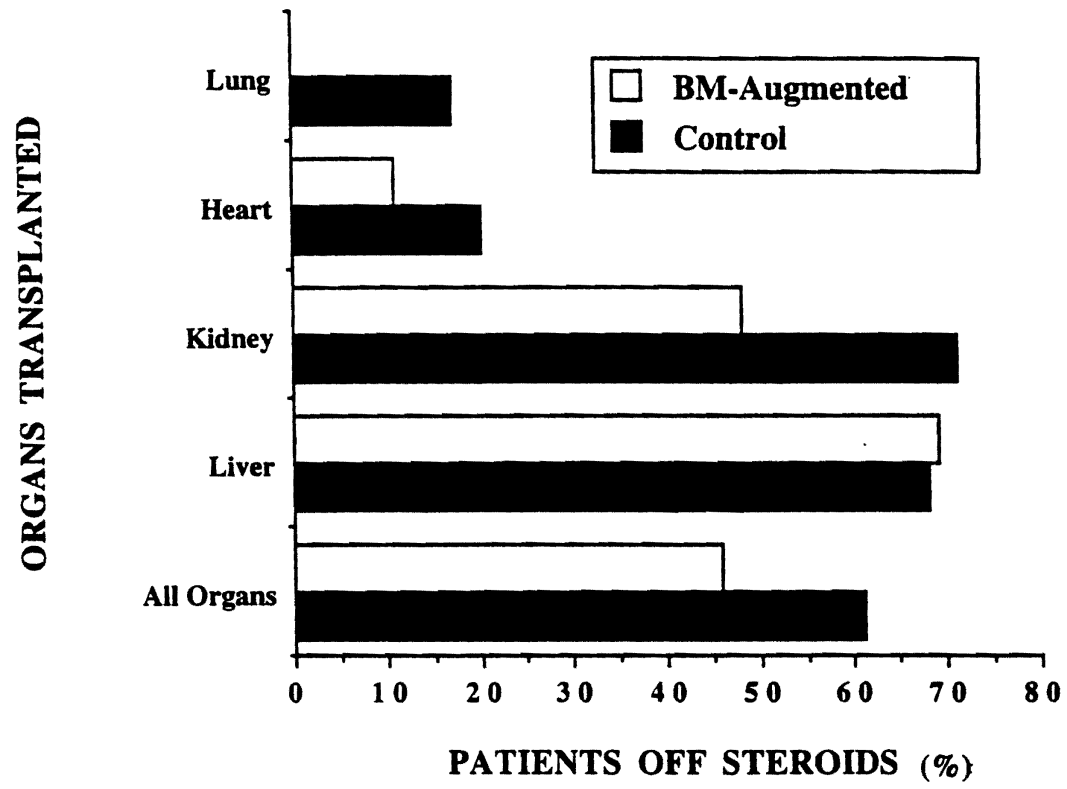

Figure 1. The incidence of steroid-free existence in BM-augmented and non-augmented (control) organ recipients who are at least 12 months post-Tx.

cipients exhibited donor-specific hypo or intermediate (DSHI) responses as compared to the controls (Figure 2). On the contrary, there was a comparable degree of immunomodulation in study and control kidney and heart recipients at a similar duration of follow-up.

\section{CONCLUSION}

Whilst in rodents and large animals, donor-specific tolerance could be achieved in months or years, the expectation of a similar outcome in humans is perhaps erroneous.

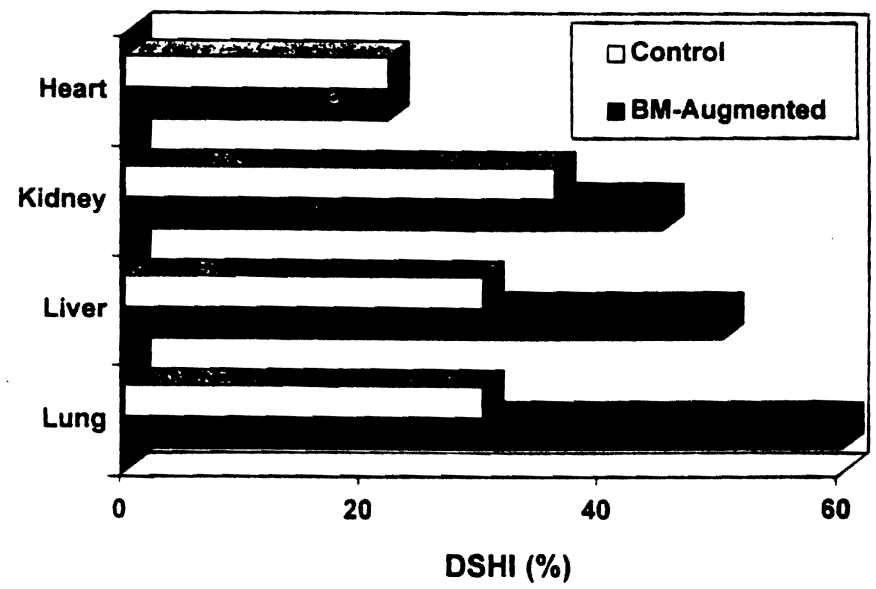

Figure 2. The incidence of evolvement of donor-specific hypo or intermediate (DSHI) reactivity determined by MLR assay in BM-augmented and control organ allograft recipients at a two-year follow-up. 
Given our experience with long-term successful organ transplant recipients who are currently being weaned off IS under a meticulously planned protocol, it is anticipated that only a select cohort of patients in this study will ever achieve a drug-free state perhaps years if not a decade after Tx.

\section{REFERENCES}

1. Snell GD. The homograft reaction. Annu Rev Microbio 1957; 11:439.

2. Steinmuller D. Immunization with skin isografts taken from tolerant mice. Science 1967; 158:127.

3. Steinmuller D. Passenger leukocytes and the immunogenicity of skin allografts. J Invest Dermatol 1980; 75:107.

4. Elkins WL, Guttmann RD. Pathogenesis of a local graft versus host reaction: Immunogenicity of circulating host leukocytes. Science 1968; 159:1250.

5. Lafferty $\mathrm{KJ}$, et al. Thyroid allograft immunogenicity is reduced after a period in organ culture. Science $1975 ; 188: 259$.

6. Silvers WK, et al. The influence of removing passenger cells on the fate of skin and parathyroid allografts. Diabetes 1982; 31:60.

7. Lacy P, Davie J. Finke E. Prolongation of islet allograft survival following in vitro culture $\left(24^{\circ} \mathrm{C}\right)$ and a single injection of ALS. Science 1979; 204:312.

8. Steinman RM. The dendritic cell system and its role in immunogenicity. Annu Rev Immunol 1991; 9:271.

9. Lechler RI, Batchelor JR. Restoration of immunogenicity to passenger cell-depleted kidney allografts by the addition of donor strain dendritic cells. J Exp Med 1979; 150:455.

10. McKenzie JL, Beard ME, Hart DJN. The effect of donor pretreatment on interstitial dendritic cell content and rat cardiac allograft survival. Transplantation 1984; 38:371.

11. Faustman DL, et al. Prevention of rejection of murine islet allografts by pretreatment with anti-dendritic cell antibody. Proc Natl Acad Sci USA 1984; 81:3864.

12. Austyn JM, Larsen CP. Migration pattern of dendritic leukocytes. Transplantation 1990; 19:1.

13. Starzl TE, et al. Cell migration and chimerism after whole organ transplantation: The basis of graft acceptance. Hepatology 1993; 17:1127.

14. Streilein JW, et al. Immunoregulatory mechanisms of the eye. J Neuroimmunol 1992; 39:185.

15. Thomas JM, et al. Further studies of veto activity in rhesus monkey bone marrow in relation to allograft tolerance and chimerism. Transplantation 1994; 57:101.

16. Qian S, et al. Murine liver allograft transplantation: Tolerance and donor cell chimerism. Hepatology 1994; 19:916.

17. Austyn JM, et al. Isolation and characterization of dendritic cells from mouse heart and kidney. J Immunol 1994; 152:2401.

18. Starl TE, et al. Cell migration, chimerism, and graft acceptance. Lancet 1992; 339:1582.

19. Starzl TE, et al. Cell migration and chimerism after whole organ transplantation: The basis of graft acceptance. Hepatology 1993; 17:1152.

20. Murase $\mathrm{N}$, et al. Variable chimerism, graft versus host disease, and tolerance after different kinds of cell and whole organ transplantation from Lewis to Brown-Norway rats. Transplantation 1995;60:158.

21. Demetris AJ, et al. Hematolymphoid cell trafficking, microchimerism, and GVHD reactions after liver, bone marrow, and heart transplantation. Transplantation Proc 1993; 25:3337.

22. Lu L. et al. Growth of donor-derived dendritic cells from the bone marrow of murine liver allograft recipients in response to granulocyte/macrophage colony-stimulating factor. J Exp Med 1995; 182:379.

23. Murase N, et al. Multilineage hematopoietic reconstitution of supralethally irradiated rats by syngeneic whole organ transplantation: With particular reference to the liver. Transplantation 1996; 61:1;

24. Rugeles MT, et al. Evidence for the presence of multilineage chimerism and progenitors of donor dendritic cells in the peripheral blood of bone marrow-augmented organ transplant recipients. J Immunol (submitted). 1996.

25. Fontes $\mathbf{P}$. et al. Bone marrow-augmentation of donor-cell chimerism in kidney, liver, heart and pancreas islet transplantation. Lancet $1994 ; 344: 151$.

26. Zeevi A, et al. Immune status of recipients following bone marrow augmented solid organ transplantation. Transplantation 1995: 59:616. 